\title{
BGaze system as a novel technology for cost effective ADHD diagnosis in public health system
}

\author{
Alba García ${ }^{1,2}$, Paloma Varela ${ }^{3}$, Josep Cañete ${ }^{3}$, Hans Supèrr,4-6 \\ University Pompeu Fabra, Barcelona, Spain; 2) Braingaze S.L., Mataró, Spain; 3) CSdM, Mataró, Spain; 4) ICREA, Barcelona, Spain; 5) Instituto de Neurociencias, \\ Barcelona, Spain; 6) Department of Cognition, Development and Educational Psychology, Faculty of Psychology, University of Barcelona, Barcelona, Spain \\ Contact hans.super@icrea.cat
}

\section{BGAZE}

BGaze system is a cloud-based platform for diagnosing ADHD from Braingaze. It includes a semi-structured interview (ACE and ACE+ from Suzy Young), rating scales, and objective marker, which is able to correctly classify ADHD patients from healthy/clinical controls with an accuracy of 92\%. BGaze protocol is designed to facilitate and standardize ADHD diagnosis. In this study we analyzed whether the use of the BGaze system can help reducing costs related to ADHD diagnosis.

\section{METHODS}

Data from Hospital Mataro and from Catalan Healthcare System were used. Only naïve children ( $N=93 ; 66 \%$ male; mean $\pm S D$ : $10,29 \pm 2,75$ years of age) were included. The cost of the current diagnostic pathway was compared to the hypothetical cost of a pathway designed to include BGaze

\section{RESULTS}

On average 4,7 hours (5-6 visits) was needed for an ADHD diagnosis. Psychologists spent $2,49 \pm 1.46 \mathrm{hrs}$ (mean $\pm S D$ ) while psychiatrist spent $0.93 \pm 0,6 \mathrm{hrs}$. In addition, $1.3 \mathrm{hrs}$ was needed for a nurse and administrative assistant. If using BGaze system, all professionals would reduce the time for an ADHD diagnosis (psychiatrist $0.9 \pm 0,6$ vs. $0.8 \pm 0,41)$, psychologist $(2.49 \pm 1.46$ vs $1.8 \pm 0,62)$, nurse $(0.3$ vs. 0,3$)$ and administrative assistant ( $1 \mathrm{~h}$ vs. $0.7 \mathrm{~h})$. This means that BGaze system could reduce the costs from $€ 101.72$ to $€ 75.95$.

\section{DISCUSSION}

We show that the use of BGaze platform reduces the time spent to diagnose ADHD, which translates into a cost saving of $25 \%$. Besides cost effectiveness, the advantage of a computerized platform is that all centers use a standardized protocol.

\section{CONCLUSIONS}

Adopting the BGaze protocol standardizes ADHD diagnosis and reduces time for professionals to establish an ADHD diagnosis.

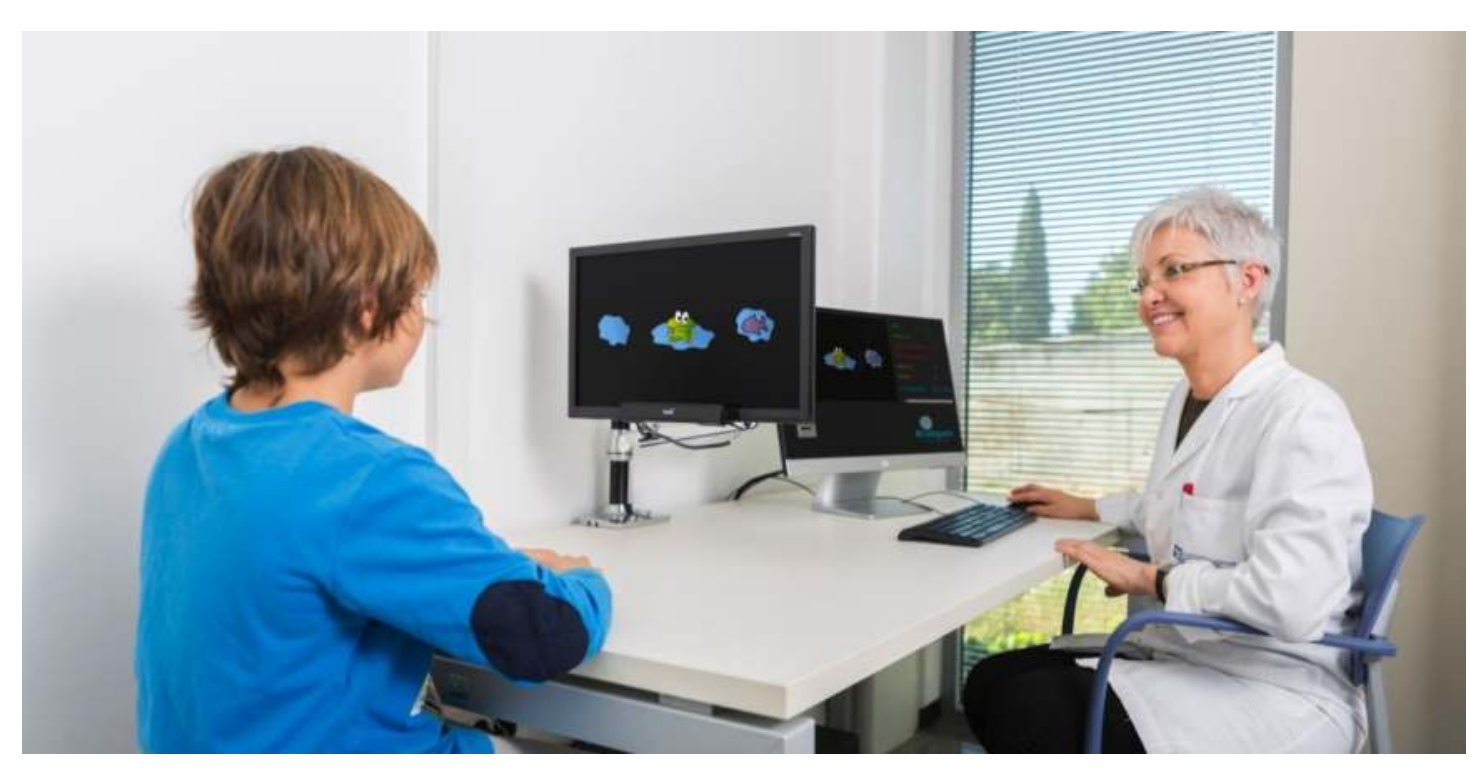

BGaze system with neural marker test for diagnosis of ADHD

Time spent for diagnoses per patient

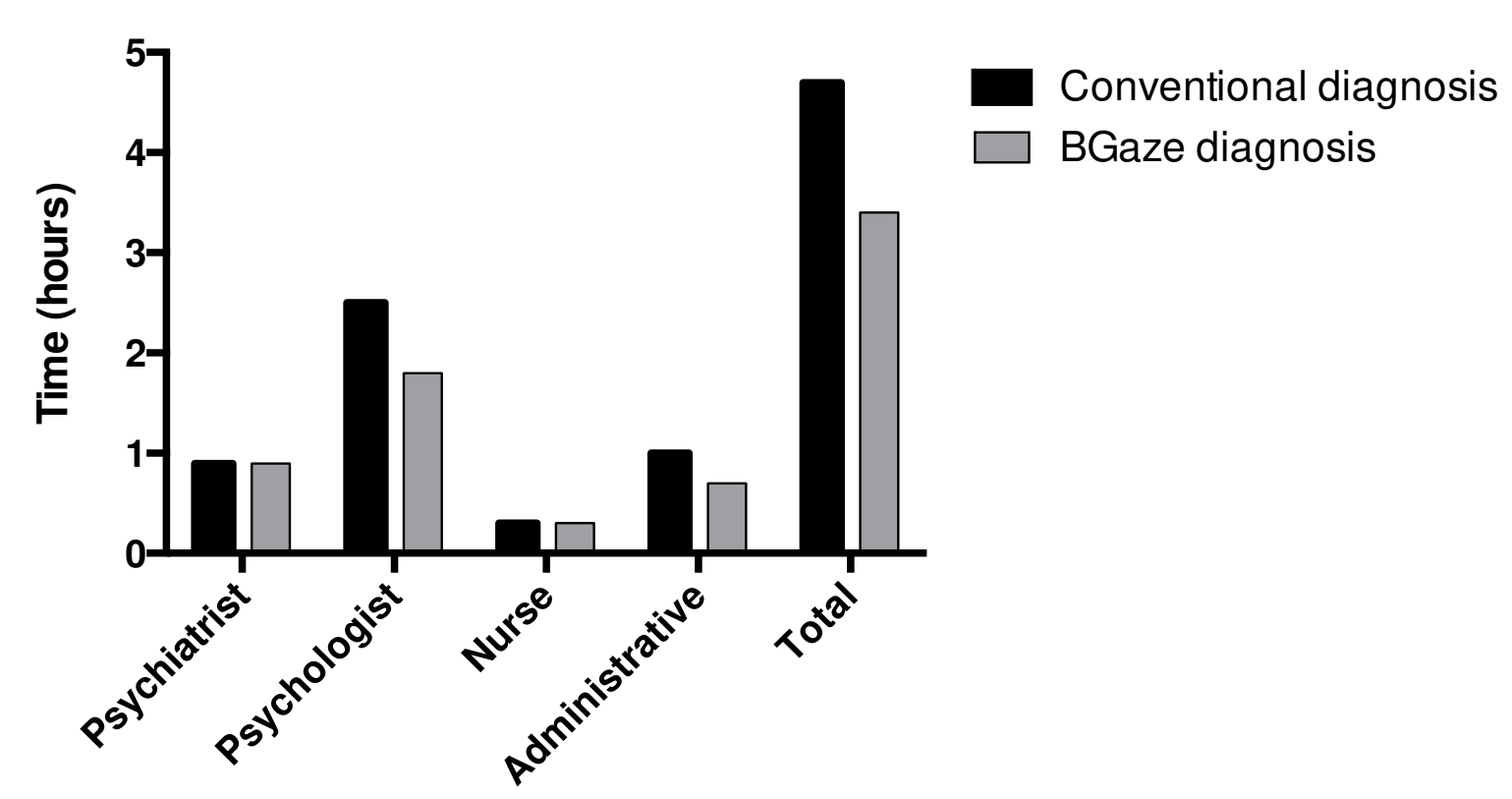

Time spent for both conventional and BGaze diagnoses per patient. Black bars represent an average of hours spent on conventional diagnosis. Grey bars represent the theoretical average of hours would be spent on BGaze diagnosis. $N=50$ (ADHD patients diagnosed by CSMIJ Mataró).

\section{Monetary costs of the diagnosis per patient (CSMIJ Mataró)}

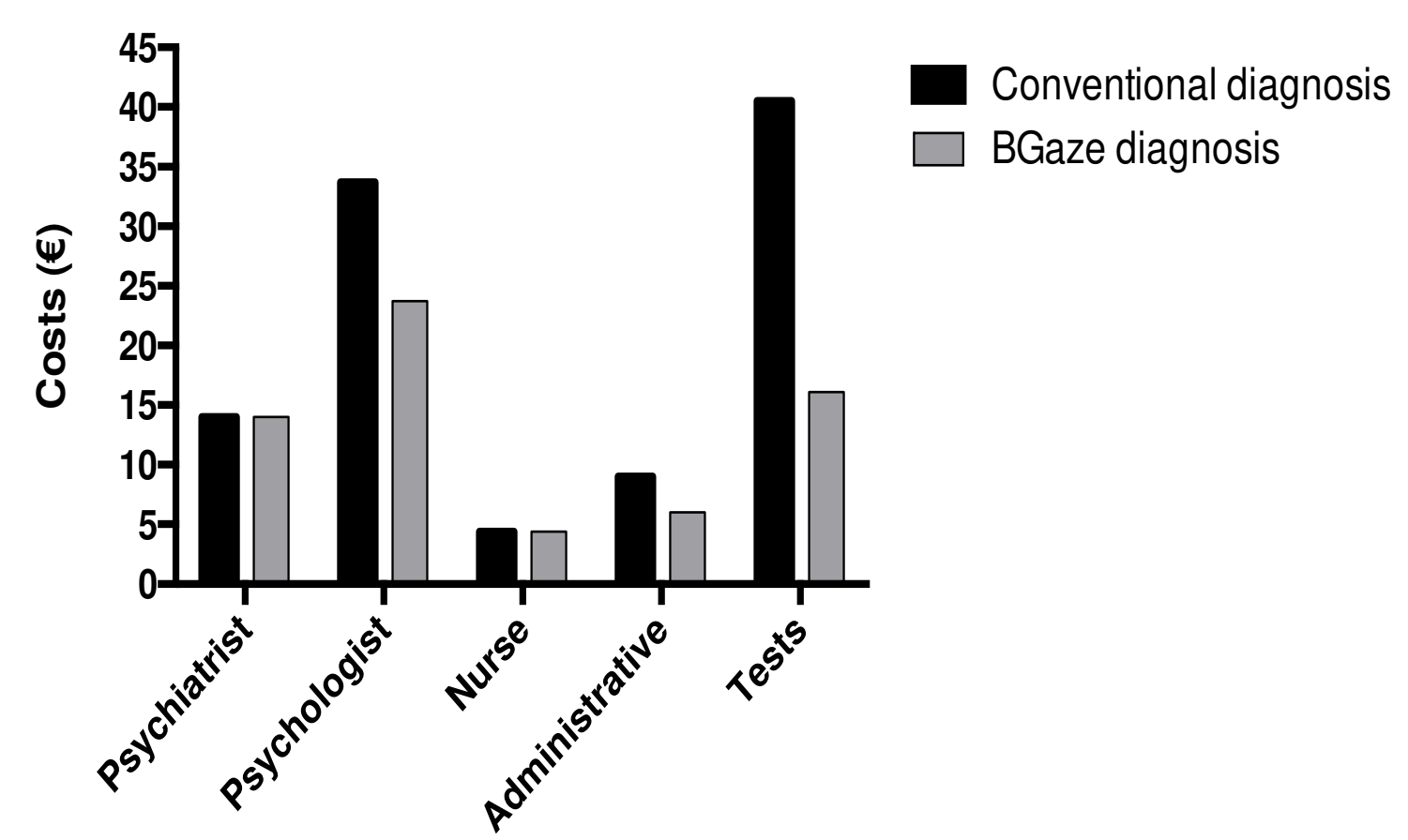

Monetary costs of both conventional and BGaze diagnoses per patient. Black bars represent an average of euros dedicated to conventional diagnosis. Grey bars represent the theoretical average of euros would be dedicated to BGaze diagnosis. $N=50$ (ADHD patients diagnosed by CSMIJ Mataró). 